\title{
Surveillance for hepatocellular cancer
}

\author{
Authors: Anna Roskilly ${ }^{A}$ and Ian A Rowe ${ }^{B}$
}

Hepatocellular carcinoma (HCC) is a common complication of cirrhosis. The incidence of HCC is rising and HCC-related mortality is rising in parallel such that there were more than 1,700 deaths in the UK in 2015. Since cirrhosis is a known risk factor for the development of HCC and early diagnosis is associated with improved outcomes, surveillance for the development of HCC using regular ultrasound scans is recommended by many expert bodies including the National Institute for Health and Care Excellence (NICE). This surveillance is not supported by high-quality evidence and there is an increasing appreciation of the associated harms. In this review the likely benefits of surveillance are discussed together with recommendations to increase the effectiveness of surveillance overall.

KEYWORDS: Hepatocellular cancer, cirrhosis, non-alcoholic fatty liver disease, liver failure, surveillance

\section{Introduction}

Hepatocellular carcinoma (HCC) is the 6th most common cancer, and the second most common cause of cancer-related mortality worldwide. ${ }^{1}$ It is recognised as a major complication of cirrhosis alongside liver failure. Among individuals with cirrhosis and no signs of liver failure, termed 'compensated cirrhosis', approximately $2-7 \%$ develop HCC annually. ${ }^{2}$ Over the past decade the incidence of HCC has been rising in the UK to a rate of 3.8 and 1.7 per 100,000 for males and females respectively. ${ }^{3}$

Globally, $\mathrm{HCC}$ is most common in patients with underlying chronic hepatitis B virus (HBV) and hepatitis C virus (HCV) infection. However, with high rates of alcohol-related liver disease (ArLD) and the growing incidence of non-alcoholic fatty liver disease (NAFLD), due to the rise in obesity and the metabolic syndrome, the incidence of HCC within the UK population is expected to continue to increase. ${ }^{4-6}$

Currently, most individuals with HCC are diagnosed at a late and incurable stage when treatment options are limited. ${ }^{7}$ In addition, the development of HCC in the setting of cirrhosis with or without liver failure inevitably increases the risks of intervention and raises concerns about treatment suitability. Consequently, mortality is

Authors: ${ }^{\text {A }}$ clinical research fellow, Liver Unit, St James's University Hospital, Leeds Teaching Hospitals NHS Trust, Leeds, UK; B university academic fellow and honorary consultant hepatologist, Institute for Biomedical and Clinical Sciences, University of Leeds, UK high in patients with cirrhosis who develop $\mathrm{HCC}^{8}{ }^{8}$ Mortality data from the UK for HCC demonstrates that this is rising in parallel with the incidence rates of HCC such that in 2015 there were approximately 1,700 deaths due to HCC.

Since cirrhosis is readily identified and the risk of HCC is known, regular surveillance testing using ultrasound scanning is proposed to identify HCC early and to facilitate curative treatment. ${ }^{9}$ This rationale is supported by many international guidelines for the management of patients with cirrhosis including the European Association for the Study of the Liver (EASL) and the American Association for the Study of Liver Diseases (AASLD), as well as by recent guidance issued by the National Institute for Health and Care Excellence (NICE). ${ }^{10-12}$ However, the recommendation that patients with cirrhosis have regular surveillance for HCC is not universally accepted and the majority of patients do not receive surveillance. $^{13-15}$

\section{The imperative for surveillance}

Many international authorities, including the AASLD and the EASL recommend that high risk groups are entered into surveillance programmes. This includes the majority of patients with cirrhosis but not those with advanced liver failure unless they are on the transplant waiting list. Patients with non-cirrhotic HBV infection and patients with HCV infection and advanced fibrosis (defined as fibrosis stage 3 [out of 4 , where 4 is cirrhosis]) are also recommended to have surveillance due to the increased incidence of HCC in these groups. ${ }^{11}$ Surveillance of individuals without cirrhosis will not be discussed further in this article.

Surveillance relies on the ideal that if we detect early asymptomatic cancers, curative treatment options are available to these patients. These treatments include resection, transplantation and radiofrequency ablation, each of which can achieve 5-year survival rates near $70 \%$ for small lesions, $<2.5 \mathrm{~cm}$ in diameter. ${ }^{9}$ Recurrent HCC is frequently observed after liver resection and ablation since the development of HCC is associated with the underlying cirrhosis and that cirrhosis is not treated or targeted by local therapies. Liver transplantation offers a real prospect of cure for these patients; however, in practice this option is limited in applicability due to comorbidity and limited in availability due to a shortage of donor organs. ${ }^{7}$

\section{Current provision of surveillance}

Reports of HCC surveillance in patients with cirrhosis consistently show poor uptake and adherence to the published guidance. A UK survey confirmed these international findings and showed that current surveillance for HCC is poor, provision is ad hoc and there 
were not standardised recall policies for follow-up of abnormal findings. ${ }^{16}$ More specifically, 131 physicians responded from 156 units across the UK. Of these, $97 \%$ stated that there was a HCC surveillance programme available in their hospital but this was not supported by later responses in the survey. Seventy-six percent stated that this was arranged on an ad hoc basis. Furthermore, only $22.6 \%$ of respondents said that ultrasonography was performed by a radiologist or radiographer with a specialist interest in liver disease or knowledge of HCC surveillance. This suggests that detection of small lesions by surveillance may not be optimal. A further issue which was highlighted by the survey was that decision making regarding which patients were deemed high risk and entered surveillance was variable between responders. Only $36.8 \%$ believed that surveillance was cost effective, although $60.1 \%$ thought that outcomes were improved. Respondents were further asked about barriers to effective surveillance and reasons included access to radiology services, lack of a liver database, doubts over effectiveness and patient adherence. Studies from the USA echo these findings and demonstrate that only a minority of patients who were at risk underwent surveillance, further questioning the effectiveness of surveillance. ${ }^{15,17}$

Critically, in the UK $30 \%$ of patients did not receive written or verbal information regarding surveillance. ${ }^{16}$ Consequently, patients are often not giving informed consent to participation and are not aware of its limitations or the frequent need for further investigations after an initial abnormal ultrasound scan. This too may have an impact on patient compliance with screening ultrasonography. ${ }^{18}$

The overall conclusion from these studies is that the provision of HCC surveillance, both in the UK and more widely, is poor. This brings into question the effectiveness of the current system of surveillance in identifying and enabling treatment of early stage HCC.

\section{Possible benefits of surveillance}

The intended benefits of entering a patient into a surveillance programme are clear. The aim is to detect early cancers in at-risk groups and enable potentially curative treatments for this group of patients. More specifically, the aim is to detect cancers which are less than $2-2.5 \mathrm{~cm}$ which can be treated with ablative strategies, as treatment in this group has demonstrated significant survival benefit.

While surveillance for HCC in patients with cirrhosis is logical, there is limited evidence from randomised controlled trials that surveillance for HCC provides survival benefit in this patient population. ${ }^{19,20}$ A single Chinese randomised controlled trial did demonstrate a survival benefit in those screened when compared to the control group. ${ }^{21}$ However, this study has been criticised due to its design and subsequent analysis, ${ }^{22}$ and it is likely to have overestimated the benefits of surveillance due to these concerns. Furthermore, this trial is not readily applicable to a Western population with cirrhosis since it included patients with HBV, mostly without cirrhosis. Consequently, it is questionable to what extent this trial can be relied upon to provide the necessary highquality evidence to support surveillance in the UK.

Two recent systematic reviews have focused on survival benefit/efficacy of 6-monthly ultrasound surveillance. ${ }^{19,20}$ These two reviews, based on the same non-randomised studies report conflicting interpretations. The first pooled the data in meta-analysis and suggested that there was clear evidence that surveillance improved survival outcomes in patients with

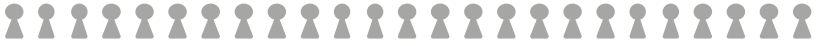

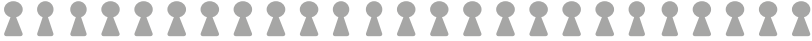

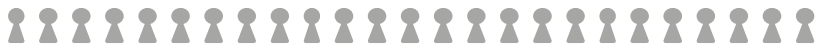

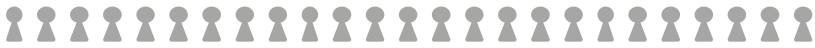

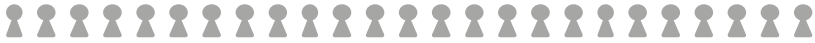

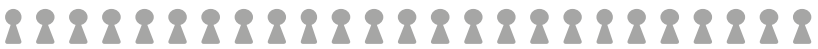

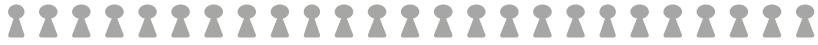
8988988989889898989898988

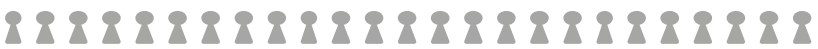

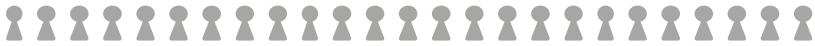
8988989889898989889898988

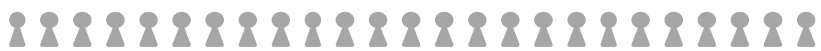
8288989889898989889898988

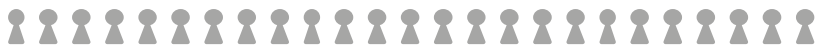

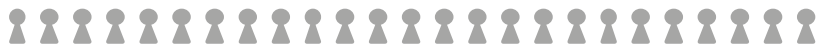

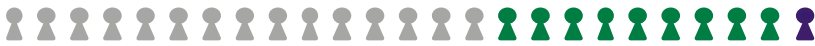

Fig 1. Illustration of the number of patients required to be in surveillance to prevent one death each year. Individuals under surveillance are illustrated. Those without hepatocellular carcinoma (HCC) are shown in grey ( $n=390)$, those developing HCC are shown in green $(n=9)$, and the individual whose death from HCC is prevented is shown in purple $(n=1)$.

cirrhosis. ${ }^{20}$ The second review did not pool data, recognising heterogeneity in study design and in the included patient populations, and concluded that while it was possible to achieve earlier diagnosis of HCC there was only low level of evidence to support a survival benefit from routine surveillance for HCC. ${ }^{19}$

The non-randomised studies summarised by the two systematic reviews do consistently show improvement in outcomes in patients with HCC detected in surveillance. The relative reduction in mortality is often quoted and is estimated at approximately $30 \%$. This only tells part of the story. The absolute risk reduction in mortality in the highest quality non-randomised studies (those that include an estimate of the effect of lead time bias) is in the region of $10 \%{ }^{13,23}$ To illustrate the impact of this, the number of patients with HCC needed to be in surveillance to prevent one death from HCC is 10. This is an underestimate of the number of patients with cirrhosis who would need to be in surveillance. Assuming an annual incidence of HCC of $2.5 \%$ then 400 patients would need to be in surveillance for $10 \mathrm{HCCs}$ to be diagnosed and for the one death to be prevented (Fig 1).

This estimate of the number needed to be in surveillance might also allow one to calculate the impact of a coordinated surveillance effort at the national level. As an indication of the likely impact of a coordinated surveillance effort, there are estimated to be in excess of 17,000 new diagnoses of cirrhosis annually in the $\mathrm{UK}^{24}$ and the number of individuals with cirrhosis is likely to be in the region of $50,000 .^{12}$ If $80 \%$ of this group were diagnosed with cirrhosis (40,000 individuals) and of these $80 \%$ were good candidates for surveillance (32,000 individuals), then with a number needed to be in surveillance of 400 to prevent one death it might be estimated that 80 deaths from HCC would be prevented as a consequence. The assumptions made here are optimistic and to implement surveillance effectively would require 
diagnosis of individuals with cirrhosis before liver failure manifests as has been recommended in the NICE cirrhosis guideline. ${ }^{12}$ Currently, only $50 \%$ of diagnoses of cirrhosis are made before the onset of liver failure ${ }^{25}$ and this indicates that the impact of surveillance today will be substantially less than the estimate above. This clearly identifies the limitations to surveillance as it currently stands.

\section{Predictable harms of surveillance}

There is debate as to the effectiveness of surveillance for HCC and its impact on overall survival rates. Evidence from randomised controlled trials is limited. Few studies focus on the harm to the patient, but instead emphasise cost implications of surveillance programmes.

When considering the risk versus benefits of HCC surveillance we must consider the possible harm to the patient. Such concerns include false-positive testing resulting in unnecessary and risk-associated procedures such as liver biopsy, overdiagnosis of HCC among patients with cirrhosis, as well as false-negative investigations resulting in delayed diagnosis of $\mathrm{HCC}^{26}$ These factors have the potential to result in both physical and psychosocial harm to a patient in surveillance. ${ }^{18,27}$ The psychosocial harms of surveillance have latterly been addressed to a limited extent in the literature. A recent report from the USA suggests that up to $75 \%$ of patients in surveillance are concerned that they will develop HCC and many of these patients are concerned that they will die from the disease. In fact, the patients included in that study had advanced liver disease and $50 \%$ had evidence of liver failure with limited life expectancy. ${ }^{18}$ Whether surveillance itself was exacerbating the concerns about the development of HCC was not investigated but in patients with already significantly impaired quality of life any adverse contribution from surveillance is clearly unwanted.

The concern regarding the risk of false-positive scans which result in further investigation with magnetic resonance imaging (MRI) and computed tomography (CT) has not been explicitly addressed. Prospective studies of surveillance ${ }^{28,29}$ suggest that up to $5 \%$ of individuals in surveillance each year have false positive tests and additional and unnecessary tests. More recently, it has been demonstrated that up to $25 \%$ of patients in surveillance programmes will experience physical harm from a false-positive or indeterminate test including further investigation with CT, MRI, angiography or liver biopsy. ${ }^{27}$ This lends perspective when we consider that only $1-5 \%$ of patients with cirrhosis will develop $\mathrm{HCC}$ and a recent modelling study suggests that many individuals entering a surveillance programme will experience harm as a result. ${ }^{30}$

A final consideration is the cost effectiveness of surveillance programmes in a healthcare system which is underfunded and under-resourced at present. Multiple cost-effectiveness studies have examined this question and have reported variable findings depending on the incidence as well as the characteristics of local health systems. ${ }^{31}$ These analyses have not considered management of patients after the first treatment of HCC and this is a significant limitation as there are appreciable rates of both HCC recurrence and progression of liver disease after treatment with resection or radiofrequency ablation; this will significantly impact future morbidity and mortality. In the recently published NICE guidance a new health economic evaluation was undertaken. It did not support surveillance at the $£ 20,000$ per quality-adjusted life year (QALY) threshold that is typically used by NICE but the recommendation for surveillance use was justified on the basis that implementation was already widespread. ${ }^{12}$

\section{Strategies for improvement}

There are many aspects of the current HCC surveillance programme that can be improved, most importantly by including those patients who stand to benefit the most and excluding those who are unlikely to benefit. It is evident that the balance of benefit and harm is dependent on the patient group and so careful selection is critical. Therefore, future guidance should detail how we define this at-risk and ideal population. Factors to consider include age - younger patients inevitably have more life years to gain from early diagnosis of HCC; stage of cirrhosis those with early cirrhosis having more time before end-stage liver disease develops; and limited medical comorbidity again increasing the likely benefit of surveillance. These factors will be key in determining those patients who will be suitable for curative treatment options should HCC be detected. In addition, these factors also identify patients at low risk of competing mortality, both from liver failure as well as from extrahepatic diseases where the benefits of surveillance may be greatest.

Critically, this strategy may not identify those at the greatest risk of developing HCC since it is individuals with very advanced liver disease who are at greatest risk. Patients with advanced liver disease are often unsuitable for any treatment for HCC other than liver transplantation and if this is not available, for whatever reason, these individuals should not be offered surveillance.

An important factor when considering patients who are thought to be potentially appropriate for surveillance is the role of informed consent, data for which are now beginning to emerge. ${ }^{30}$ Consideration also needs to be given to the feasibility of implementing surveillance programmes including a specialist nursing support, dedicated ultrasound scan lists and same-day clinics, alongside maintaining a database of eligible patients with cirrhosis. There also needs to be a system in place for recall of these patients and management of abnormal test results which is currently provided on an ad hoc basis.

Although there is a rationale for HCC surveillance, clinicians should take care in selecting patients for these programmes while evaluating risks versus benefits and considering patient wishes. This should enable implementation of a safe and effective surveillance programme UK wide, ensuring that those who stand to benefit most are included and surveyed appropriately in a costeffective manner.

\section{References}

1 GBD 2013 Mortality and Causes of Death Collaborators. Global, regional, and national age-sex specific all-cause and cause-specific mortality for 240 causes of death, 1990-2013: a systematic analysis for the Global Burden of Disease Study 2013. Lancet 2015;385:117-71.

2 Forner A, Llovet JM, Bruix J. Hepatocellular carcinoma. Lancet 2012:379:1245-55.

3 Ladep NG, Khan SA, Crossey MM et al. Incidence and mortality of primary liver cancer in England and Wales: changing patterns and ethnic variations. World J Gastroenterol 2014:20:1544-53.

4 Sheron N. Alcohol and liver disease in Europe - Simple measures have the potential to prevent tens of thousands of premature deaths. J Hepatol 2016;64:957-67. 
5 Younossi ZM, Koenig AB, Abdelatif D et al. Global epidemiology of nonalcoholic fatty liver disease-Meta-analytic assessment of prevalence, incidence, and outcomes. Hepatology 2016;64:73-84.

6 Younossi ZM, Otgonsuren M, Henry L et al. Association of nonalcoholic fatty liver disease (NAFLD) with hepatocellular carcinoma (HCC) in the United States from 2004 to 2009. Hepatology 2015;62:1723-30.

7 Bruix J, Gores G], Mazzaferro V. Hepatocellular carcinoma: clinical frontiers and perspectives. Gut 2014;63:844-55.

8 Park JW, Chen M, Colombo M et al. Global patterns of hepatocellular carcinoma management from diagnosis to death: the BRIDGE Study. Liver Int 2015;35:2155-66.

9 Bruix J, Reig M, Sherman M. Evidence-based diagnosis, staging, and treatment of patients with hepatocellular carcinoma. Gastroenterology 2016;150:835-53.

10 Bruix J, Sherman M, American Association for the Study of Liver Diseases. Management of hepatocellular carcinoma: an update. Hepatology 2011;53:1020-2.

11 European Association For The Study Of The Liver, European Organisation For Research And Treatment Of Cancer. EASL-EORTC clinical practice guidelines: management of hepatocellular carcinoma. J Hepatol 2012;56:908-43.

12 National Institute for Health and Care Excellence. Clinical Guideline 50: Cirrhosis in over 16s: Assessment and management. NICE, 2016. www.nice.org.uk/guidance/ng50/evidence/full-guideline-2546537581 [Accessed 12 July 2016].

13 Mittal S, Kanwal F, Ying ] et al. Effectiveness of surveillance for hepatocellular carcinoma in clinical practice: A United States cohort. J Hepatol 2016;65:1148-54.

14 Singal AG, Nehra M, Adams-Huet B et al. Detection of hepatocellular carcinoma at advanced stages among patients in the HALT-C trial: where did surveillance fail? Am J Gastroenterol 2013;108:425-32.

15 Singal AG, Yopp A, Skinner C et al. Utilization of hepatocellular carcinoma surveillance among American patients: a systematic review. J Gen Intern Med 2012;27:861-7.

16 Cross TJS, Villanueva A, Shetty S et al. A national survey of the provision of ultrasound surveillance for the detection of hepatocellular carcinoma Frontline Gastroenterol 2016;7:82-9.

17 Davila JA, Henderson L, Kramer JR et al. Utilization of surveillance for hepatocellular carcinoma among hepatitis $C$ virus-infected veterans in the United States. Ann Intern Med 2011;154:85-93.

18 Farvardin S, Patel J, Khambaty M et al. Patient-reported barriers are associated with lower hepatocellular carcinoma surveillance rates in patients with cirrhosis. Hepatology 2017;65:875-84.

19 Kansagara D, Papak J, Pasha AS et al. Screening for hepatocellular carcinoma in chronic liver disease: a systematic review. Ann Intern Med 2014;161:261-9.
20 Singal AG, Pillai A, Tiro J. Early detection, curative treatment, and survival rates for hepatocellular carcinoma surveillance in patients with cirrhosis: a meta-analysis. PLoS Med 2014;11:e1001624.

21 Zhang BH, Yang BH, Tang ZY. Randomized controlled trial of screening for hepatocellular carcinoma. J Cancer Res Clin Oncol 2004;130:417-22.

22 Lederle FA, Pocha C. Screening for liver cancer: the rush to judgment. Ann Intern Med 2012;156:387-9.

23 van Meer S, de Man RA, Coenraad MJ et al. Surveillance for hepatocellular carcinoma is associated with increased survival: Results from a large cohort in the Netherlands. J Hepatol 2015;63:115663

24 Ratib S, West J, Crooks C], Fleming KM. Diagnosis of liver cirrhosis in England, a cohort study, 1998-2009: a comparison with cancer. Am J Gastroenterol 2014;109:190-8.

25 Ratib S, Fleming KM, Crooks C], Aithal GP, West J. 1 and 5 year survival estimates for people with cirrhosis of the liver in England, 1998-2009: a large population study. J Hepatol 2014;60:282-9.

26 Heleno B, Thomsen MF, Rodrigues DS, Jorgensen KJ, Brodersen J. Quantification of harms in cancer screening trials: literature review. BMJ 2013;347:f5334.

27 Atiq O, Tiro J, Yopp AC et al. An assessment of benefits and harms of hepatocellular carcinoma surveillance in patients with cirrhosis. Hepatology 2016;65:1196-205.

28 Trinchet JC, Bourcier V, Chaffaut C et al. Complications and competing risks of death in compensated viral cirrhosis (ANRS CO12 CirVir prospective cohort). Hepatology 2015;62:737-50.

29 Trinchet JC, Chaffaut C, Bourcier V et al. Ultrasonographic surveillance of hepatocellular carcinoma in cirrhosis: a randomized trial comparing 3-and 6-month periodicities. Hepatology 2011;:54:1987-97.

30 Taylor EJ, Jones RL, Guthrie JA, Rowe IA. Modelling the benefits and harms of surveillance for hepatocellular carcinoma: information to support informed choices. Hepatology 2017;66:1546-55.

31 Cucchetti A, Cescon M, Erroi V, Pinna AD. Cost-effectiveness of liver cancer screening. Best Pract Res Clin Gastroenterol 2013;27:96172.

Address for correspondence: Dr Ian A Rowe, Room 6.1, Clinical Sciences Building, University of Leeds, St James's University Hospital, Beckett Street, Leeds LS9 7TF, UK.

Email: i.a.c.rowe@leeds.ac.uk 